\title{
Applications of Framework in Health Care: A Survey
}

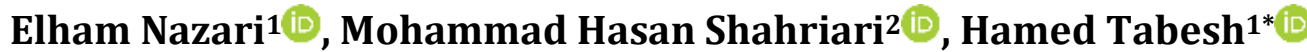

${ }^{1}$ Department of Medical Informatics, Faculty of Medicine, Mashhad University of Medical Sciences, Mashhad, Iran. ${ }^{2}$ Department of Electrical Engineering, Faculty of Computer Engineering, University of Isfahan, Isfahan, Iran.

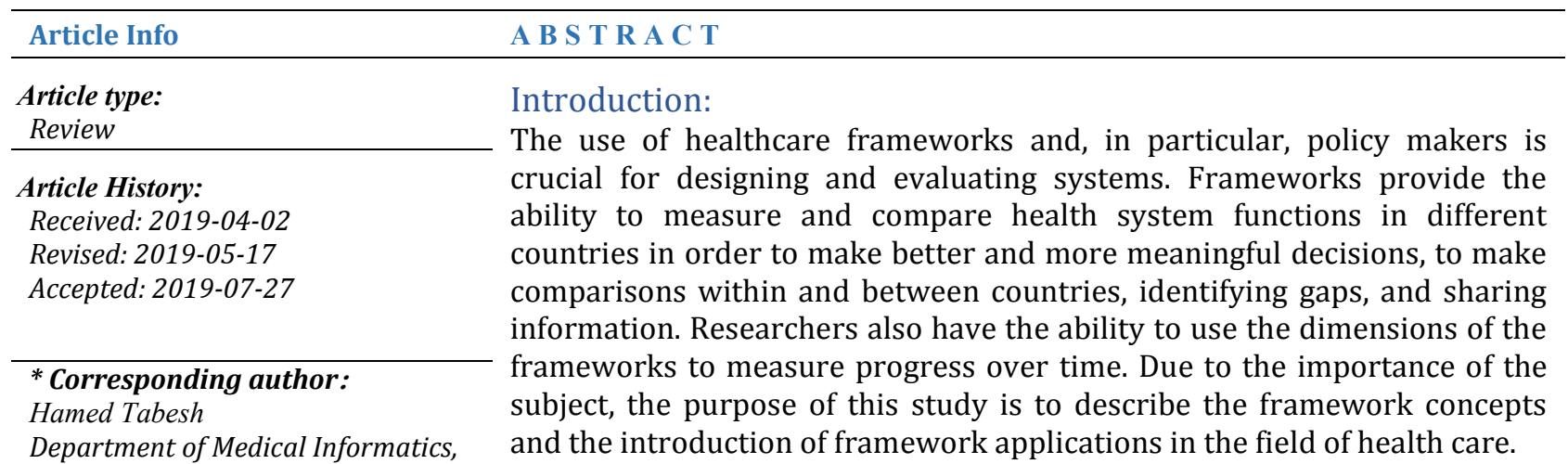

Faculty of Medicine, Mashhad

University of Medical Sciences,

Mashhad, Iran.

Email: tabeshh@mums.ac.ir

\begin{abstract}
Material and Methods:
This study is based on a search of the ProQuest, PubMed, Google Scholar, Science Direct, Scopus, IranMedex, Irandoc, Magiran, ParsMedline and Scientific Information Database (SID) databases, as well as the study of specialized keyword web sites and the standard was done. After a thorough study, 50 sources were selected according to the study objectives and were used to formulate the final article.
\end{abstract}

\section{Results:}

The framework can be used to manage health system investments, identify important research areas in the field of health, and define new and useful research.

\section{Conclusion:}

Given the importance of the health framework, the need to provide a framework for other critical health care sectors is essential.

Keywords:

Software Engineering, Framework, Healthcare.

\section{How to cite this paper}

Nazari E, Shahriari MH, Tabesh H. Applications of Framework in Health Care: A Survey. Front Health Inform. 2019; 8(1): e16. DOI: $\underline{10.30699 / f h i . v 8 i 1.186}$

\section{INTRODUCTION}

The framework focuses on one thing why and how it should work; in fact, it provides the user with a map; it is a prefabricated template or pattern that manages most common and duplicate features and the possibility provides reuse $[\underline{1}, \underline{2}]$. In other words, a framework is a basic conceptual framework that is used to solve complex problems and is usually a set of tools, materials, or components []ㅡ. This is a phrase from the architecture world to software and sometimes managed [4]. It has applications for improving and redesigning processes and services, designing and manufacturing systems, designing/ revising an enterprise architecture, integrating systems and e-services, designing new services/ products, and incorporating many benefits, including simplicity, high performance, integration ability, higher security, the ability to develop, facilitate program updates, the ability to reuse written codes $[\underline{5}, \underline{6}]$.

Frameworks have different types, including theoretical frameworks, conceptual frameworks, operational frameworks, and software frameworks. Theoretical frameworks deal with the relationships between variables such as independent, dependent, interferential and transformative variables, helping to create such frameworks in establishing and constructing hypotheses, measuring them as well as completing a researcher's perception (research question) []. The theoretical framework statement is usually used in quantitative research. While the 
researcher uses a theory or a combination of several theories to prepare a road map and explain research hypotheses. In a study, a theoretical framework for coordination care in chronic diseases requiring longterm care was presented. This framework included the association of the team members with the patient for proper interaction, team features including cultural characteristics, workload, work complexity, etc. [8]. In a study, the presentation of a theoretical framework for Public Health Ethics includes the production of benefits, avoiding, preventing, removing harms, and utility, distributive justice, respecting autonomous choices and actions, protecting privacy and confidentiality, keeping promises and commitments, disclosing information, speaking truthfully, building and maintaining trust [9].

A conceptual framework is a set of general goals and related basics that determine overall goals and objectives, and defines the basics and concepts for achieving these goals. These concepts are tips for choosing events, deals, and conditions to be

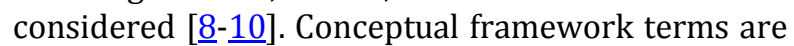
commonly used in qualitative research. The researcher uses the analysis and categorization of previous research findings as a road map for designing research tools and research questions. In studies of conceptual frameworks, for the analysis of health care organizations' performance, for performance of healthcare, including effectiveness, quality and improvement, for measuring the performance of the public health system, for quality of care, including the dimensions of efficacy, effectiveness, efficiency, empathy, and environment, for developing a comprehensive e-health assessment tool, for the IOT-based health care system using cloud computing, and the theoretical frameworks in this regard to determine good health status in Jamaica, And for supporting research on health service innovation [11-20].

The operational framework includes guiding principles, specific criteria, steps, methods and attributes that relevant to corporate policies, goals, standards, procedures and training. Generally, it describes the corporate organization or management structure. It includes how the leaders manage the company and its hierarchy of divisions or management teams. It includes policy makers of the company. It can include a guide to the principles of behavior, recruitment and promotion [21]. In a study, an operational framework for health policy included process, actor, content and context items [22]. A community-based care for mothers and infants in Queensland was also presented in the form of an operational framework, in the form of essential roles and dimensions [23]. The proposed operational framework for risk management involves injury training, athlete monitoring, risk factors, sports requirements, athlete profiles, athlete management, which can be helpful for practitioners in managing risk of injury [녀] .

The software framework is a framework for helping startup programs, and by putting all of the software components together, it is possible to develop a project or system. They can include support programs, compilers, libraries, tools and APIs [ [4]. A study was designed to use practical statistical frameworks for recommending medication to diabetic patients [25]. A study was performed using panel ensemble techniques to determine benign or malignant breast cancer [26]. A study was developed using probabilistic frameworks that processed mammography images for cancer diagnosis [27].A framework study presented using the Bayesian method for determining variations in EEG / FMRI [28].

\section{MATERIALS AND METHODS}

This review study is based on a search of the ProQuest, PubMed, Google Scholar, Science Direct, Scopus, IranMedex, Irandoc, Magiran, ParsMedline, and Scientific Information Database (SID) databases, as well as web site reviews.

Related Keywords and the standard were done. After detailed study, the full text of 50 articles, books and scientific text (derived from the website) was selected according to the objectives of the study and was used in the compilation of the final article.

\section{RESULTS}

Examples of useful health care frameworks are as follows:

\section{Framework for health care control and planning}

A $4 \times 4$ framework for health care control and planning has been proposed by Hall (Fig 1).

\section{Applied conceptual architecture of health care system using big data analysis}

An applied conceptual architecture of the healthcare system using big data analysis has been proposed by Raghupathi and Raghupathi [29], as presented in Fig 2. The proposed framework comprises several layers:

\section{Data source layer:}

Internal and external health care data sources found in a several situations in a variety of formats.

\section{Transition layer:}

This layer performs operations such as extracting, transferring, and loading data within a large data platform through data staging techniques such as 
middleware and data warehousing operations.

\section{Big data platform layer:}

This layer comprises various Hadoop ecosystem tools implemented using the Map-Reduce programming model based on the Hadoop Distributed File System (HDFS).

\section{Analysis layer:}

Performs various operations such as querying, reporting, online analytical processing and data mining techniques.

Raghupathi and Raghupathi [29] have introduced health data meta-analysis tools and platforms (Table 1).

\begin{tabular}{|c|c|c|c|c|}
\hline & Medical planning & $\begin{array}{c}\text { Resource capacity } \\
\text { planning }\end{array}$ & Materials planning & Financial planning \\
\hline Strategic & $\begin{array}{l}\text { Research, development of } \\
\text { medical protocols }\end{array}$ & $\begin{array}{l}\text { Case mix planning, } \\
\text { capacity dimensioning, } \\
\text { workforce planning }\end{array}$ & $\begin{array}{l}\text { Supply chain and } \\
\text { warehouse design }\end{array}$ & $\begin{array}{l}\text { Investment plans, } \\
\text { contracting with insurance } \\
\text { companies }\end{array}$ \\
\hline Tactical & $\begin{array}{l}\text { Treatment selection, } \\
\text { protocol selection }\end{array}$ & $\begin{array}{l}\text { Block planning, staffing, } \\
\text { admission planning }\end{array}$ & $\begin{array}{l}\text { Supplier selection, } \\
\text { tendering }\end{array}$ & $\begin{array}{l}\text { Budget and cost } \\
\text { allocation }\end{array}$ \\
\hline $\begin{array}{c}\text { Offline } \\
\text { operational }\end{array}$ & $\begin{array}{l}\text { Diagnosis and planning } \\
\text { of an individual treatment }\end{array}$ & $\begin{array}{l}\text { Appointment scheduling, } \\
\text { workforce scheduling }\end{array}$ & $\begin{array}{l}\text { Materials purchasing, } \\
\text { determining order sizes }\end{array}$ & $\begin{array}{l}\text { DRG billing, cash flow } \\
\text { analysis }\end{array}$ \\
\hline $\begin{array}{c}\text { Online } \\
\text { operational }\end{array}$ & $\begin{array}{l}\text { Triage, diagnosing } \\
\text { emergencies and } \\
\text { complications }\end{array}$ & $\begin{array}{l}\text { Monitoring. } \\
\text { emergency } \\
\text { coordination }\end{array}$ & $\begin{array}{l}\text { Rush ordering, inventory } \\
\text { replenishing }\end{array}$ & $\begin{array}{l}\text { Billing complications and } \\
\text { changes }\end{array}$ \\
\hline
\end{tabular}

Fig 1: An example of the application of a health care control and planning framework for a hospital [푸]

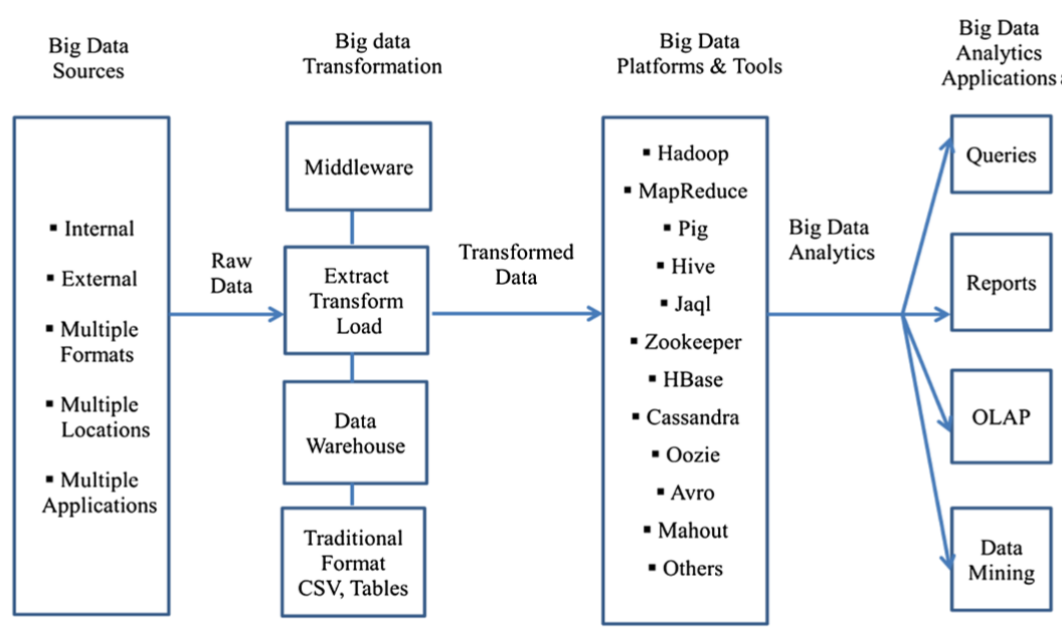

Fig 2: A conceptual framework for big data analysis [을

Table 1: Big economic data analysis tools and platform [루]

\begin{tabular}{|l|l|}
\hline Platform/Tool & Description \\
\hline The Hadoop Distributed File & $\begin{array}{l}\text { HDFS enables the underlying storage } \\
\text { for the Hadoop cluster. It divides the } \\
\text { data into smaller parts and distributes } \\
\text { it across the various servers/nodes. }\end{array}$ \\
\hline MapReduce & $\begin{array}{l}\text { MapReduce provides the interface for } \\
\text { the distribution of sub-tasks and the } \\
\text { gathering of outputs. When tasks are } \\
\text { executed, MapReduce tracks the } \\
\text { processing of each server/node. }\end{array}$ \\
\hline $\begin{array}{l}\text { PIG and PIG Latin (Pig and } \\
\text { PigLatin) }\end{array}$ & $\begin{array}{l}\text { Pig programming language is } \\
\text { configured to assimilate all types of } \\
\text { data (structured/unstructured, etc.). It } \\
\text { is comprised of two key modules: the } \\
\text { language itself, called PigLatin, and the }\end{array}$ \\
\hline
\end{tabular}




\begin{tabular}{|c|c|}
\hline Platform/Tool & Description \\
\hline & $\begin{array}{l}\text { runtime version in which the PigLatin } \\
\text { code is executed. }\end{array}$ \\
\hline Hive & $\begin{array}{l}\text { Hive is a runtime Hadoop support } \\
\text { architecture that leverages Structure } \\
\text { Query Language (SQL) with the } \\
\text { Hadoop platform. It permits SQL } \\
\text { programmers to develop Hive Query } \\
\text { Language (HQL) statements akin to } \\
\text { typical SQL statements. }\end{array}$ \\
\hline Jaql & $\begin{array}{l}\text { Jaql is a functional, declarative query } \\
\text { language designed to process large } \\
\text { data sets. To facilitate parallel } \\
\text { processing, Jaql converts "'high-level' } \\
\text { queries into 'low-level' queries" } \\
\text { consisting of MapReduce tasks. }\end{array}$ \\
\hline Zookeeper & $\begin{array}{l}\text { Zookeeper allows a centralized } \\
\text { infrastructure with various services, } \\
\text { providing synchronization across a } \\
\text { cluster of servers. Big data analytics } \\
\text { applications utilize these services to } \\
\text { coordinate parallel processing across } \\
\text { big clusters. }\end{array}$ \\
\hline Hbase & $\begin{array}{l}\text { HBase is a column-oriented database } \\
\text { management system that sits on top of } \\
\text { HDFS. It uses a non-SQL approach. }\end{array}$ \\
\hline
\end{tabular}

\section{Patient-centered personalized health care frameworks}

Chawla and Davis [31] presented a patient-centric personalized health care framework based on the collaborative refinement approach. In this framework, patient similarities are collected and personalized disease risk profiles are created for each individual. They have developed a system called Collaborative Assessment and Recommendation (CARE) to predict personalized disease risk (Fig 3).

\section{Big Data Analytical Framework Using Comprehensive Health Care System}

A big data analytical framework utilizing a comprehensive health care system has been proposed by Kim et al. (Fig 4). Vital signs (such as ECGs) extracted from the accelerometer are analyzed by the framework to provide health care services. It uses an open standard platform to support interaction between data and different devices.

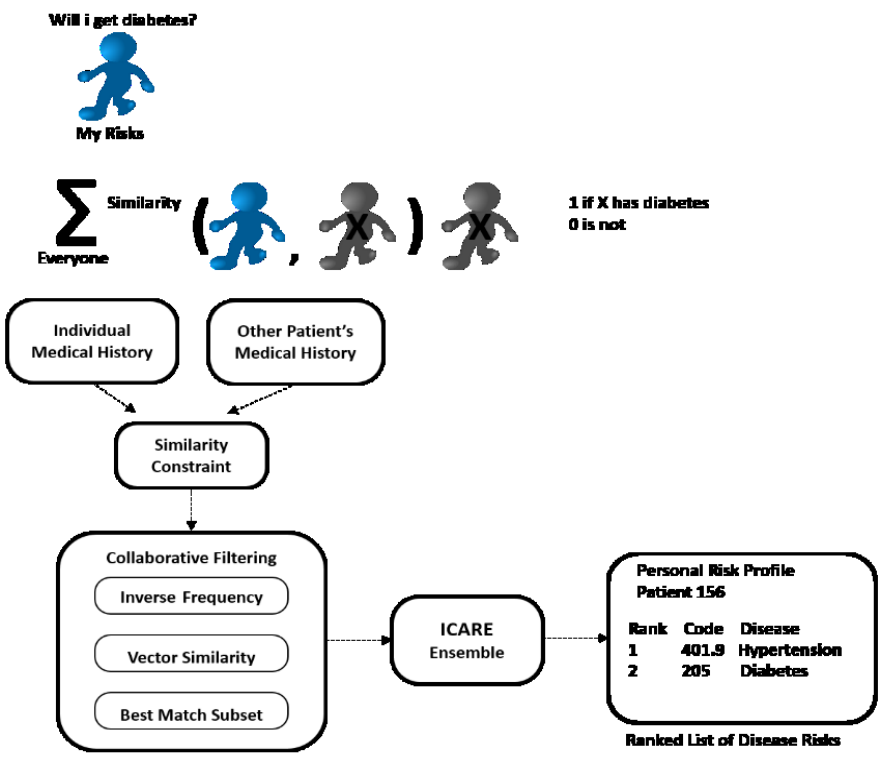

Fig 3: CARE process diagram [31] 


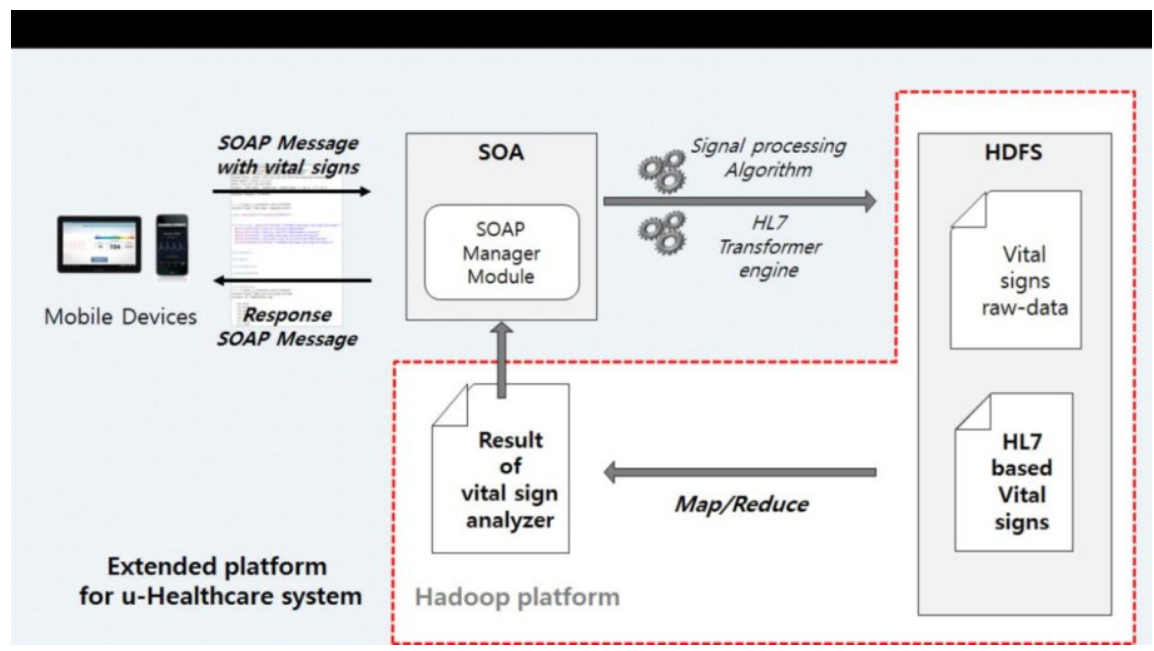

Fig 4: Big data platform for vital signs analysis [르]

\section{Health Informatics Processing Pipeline Framework}

A framework called "health informatics processing pipeline framework" has been proposed by Fang et al. [33]. As shown in Fig 5, this framework combines a sequence of steps to collect meaningful patterns of healthcare metadata. Each step in the pipeline plays a vital role in delivering valuable and high quality data analytics gains.

The pipeline steps are:

- Capturing: Defining data sources such as: electronic health care data, decision support data sources, and laboratory results

- Storing: Determining the effective storage infrastructure for analyzing health care data

- Sharing: Secure exchange of health care information between scientists and physicians

- Analyzing: Performing tasks such as data pre-processing, feature selection and machine learning

- Searching: Extracting meaningful patterns of interest from analysis results
- Decision Support: Model-Based Benefits for Effective Decision Making in the Health Informatics

6. Framework for big analysis of healthcare data in mobile cloud computing

A framework for big analysis of health care data in mobile cloud computing has been proposed by Youssef [ $\underline{34}]$ as follows (Fig 6):

- Cloud: Hosts patient information and offers health care services.

- $\quad$ EHR: Integrates patient information records from various sources such as: pharmacy, hospital, and laboratory.

- Security: Ensures security and privacy by encryption and authentication techniques.

- Big Data Analytics: Deploying different analytics tools to discover different patterns of EHR

- Care Delivery Organization (CDO): Health care delivery organizations that are distributed in different locations.

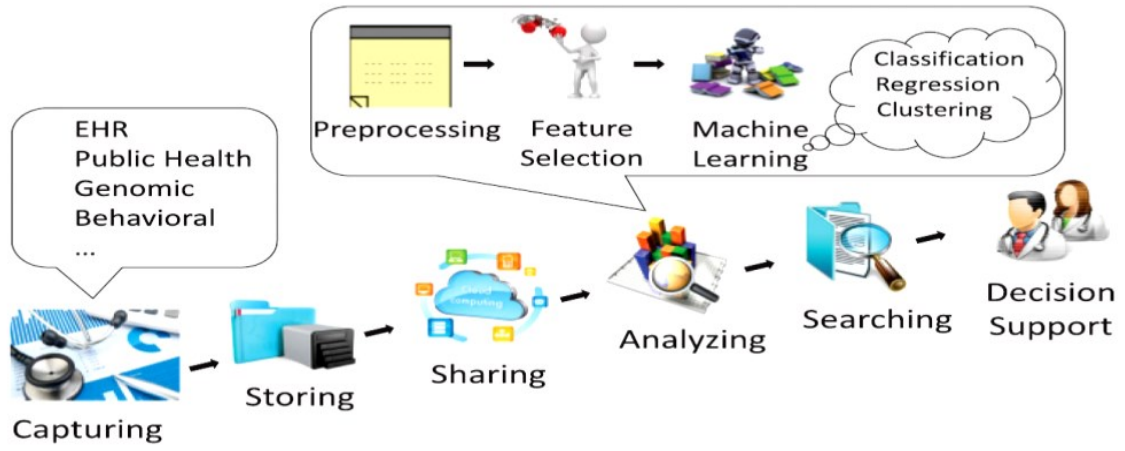

Fig 5: Pipeline health informatics processing [프] 


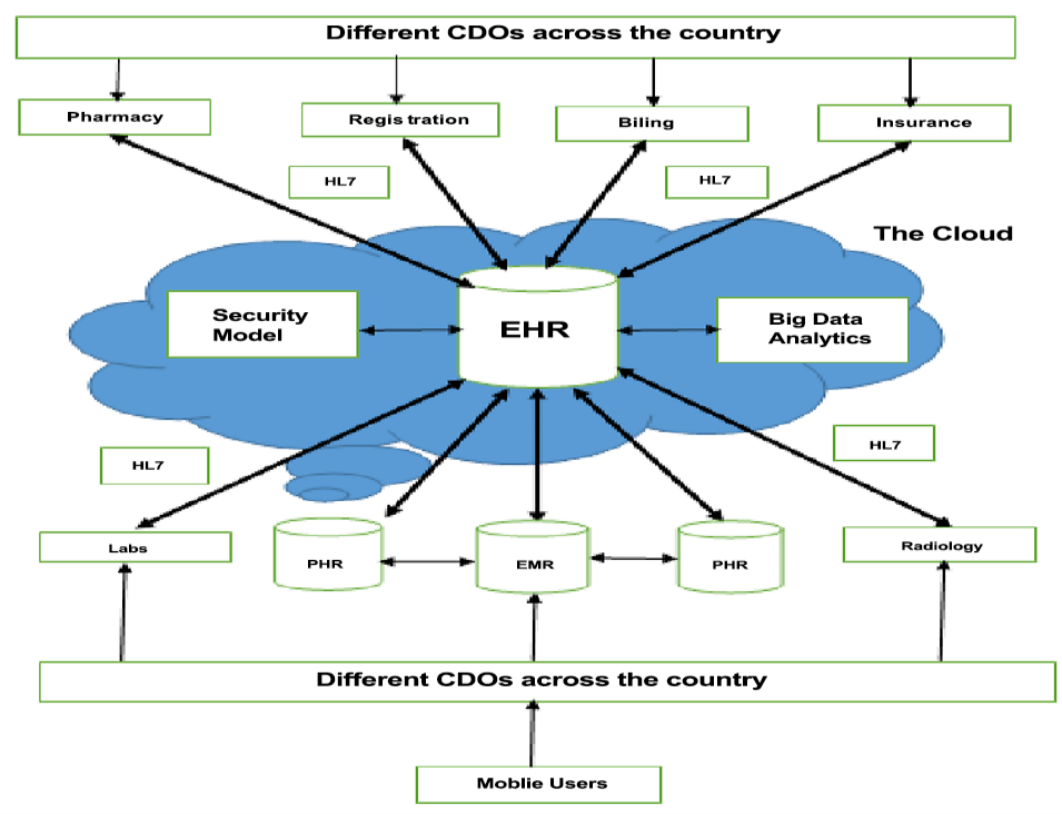

Fig 6: Framework for EHR based mobile cloud computing and big data analysis [ㅁ5]

\section{Web based ontology language framework}

The importance of semantic interaction between clinical information has been proposed by LegazGarca et al. To determine the semantic integration of Health Care Electronic Data (EHR), a Web Ontology Language (OWL) framework called ArchMS has been proposed. The data collected from relational databases to construct the ontology are converted to OWL and then the anthology built to explore data such as EHR-based classification and visualization, as presented in Fig 7.

\section{SmartHealth framework}

A physical-cyber system based on the Health Care Framework "SmartHealth Framework" was proposed by Sakr and Elgammal [36]. It integrates sensing technologies, cloud computing, IoT, and big data analytics.

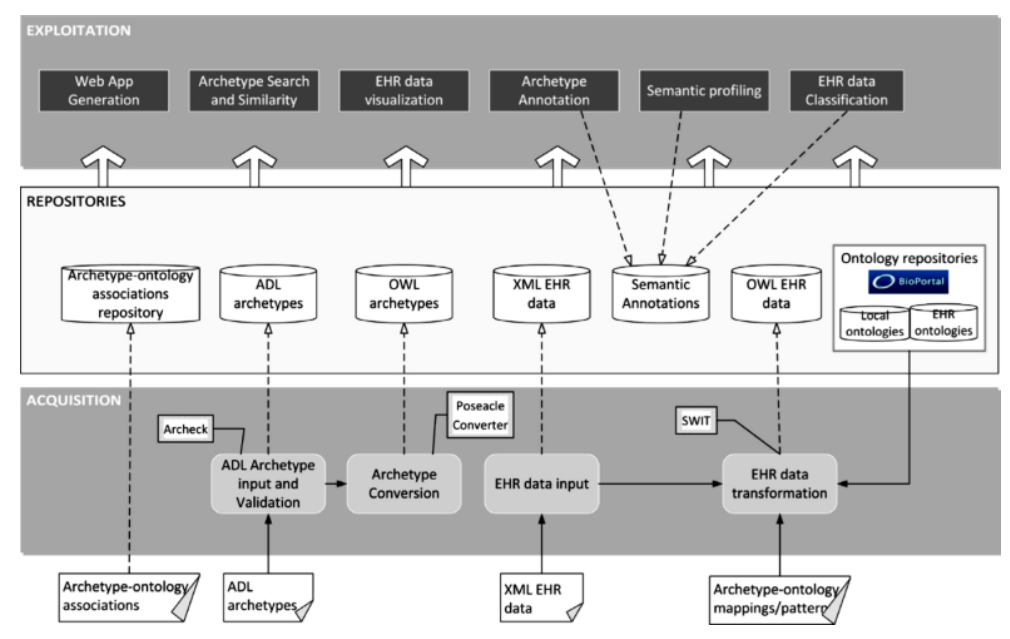

Fig 7: ArchMS architecture overview [푸] 


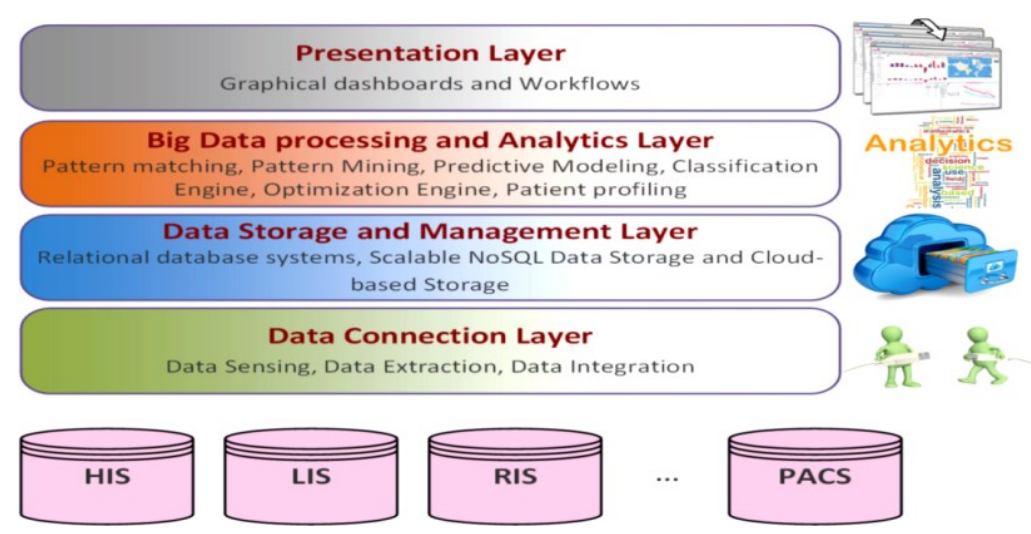

Fig 8: Layer of architecture for smart health framework [36]

As shown in Fig 8, the different layers of the SmartHealth framework are:

\section{Data Connection layer:}

For data measurement, data extraction and data integration

\section{Data Storage Layer:}

For storing relational, non-relational and cloudbased data

\section{Big data processing and analytical layer:}

Performing various analytics such as descriptive, predictive and prescriptive

\section{Presentation Layer:}

Designing dashboards and graphics workflows

\section{Cloud-based framework for big data:}

A cloud-based framework for identifying the impact of socioeconomic, geographic and demographic conditions on public health has been proposed by Mahmud et al. [37]. The framework uses the Amazon Web-based cloud platform along with geographic information system to collect, store and visualize large data.

\section{Integrated big data framework:}

Jokonya et al. [38] proposed an integrated large data framework to help control and prevent diseases such as HIV/AIDS (Human Immunodeficiency Viruses/Acquired Immune Deficiency Syndrome), Tuberculosis, and Silicosis (abbreviated as HATS). This framework addresses the need for a statistical prevention model to predict and control these diseases. Fig 9 shows the suggested framework.

\section{RFID-based healthcare framework}

The interest in RFID (Radio-Frequency Identification) technology in the healthcare industry has increased. This technology is used for such things as tracking medical equipment, hospital supplies, patient information and medications. While this technology has important implications, it raises privacy concerns. Rahman et al. [39] proposed an RFID-based healthcare framework, PriSens-HSAC, to address privacy issues. As shown in Fig 10, this framework contains two components:

\section{PriSens Protocol:}

Used as an authentication protocol to measure RFID tags.

HSAC:

Provides a mechanism for accessing the health care service to ensure user privacy.

12. Cloud-based distributed health information system framework with emphasis on security and privacy aspects:

A cloud-based distributed health information system framework with emphasis on security and privacy aspects has been proposed by Sarkar [40]. The framework proposes a set of security constraints and access control mechanisms to ensure the integrity, confidentiality, and privacy of medical data (Fig 11). 


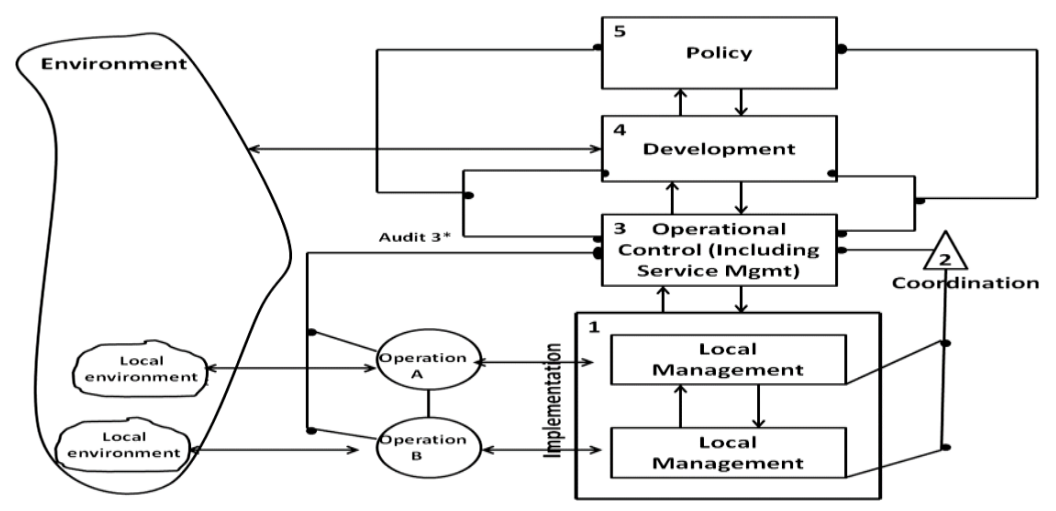

Fig 9: HATS metadata conceptual framework [묘]

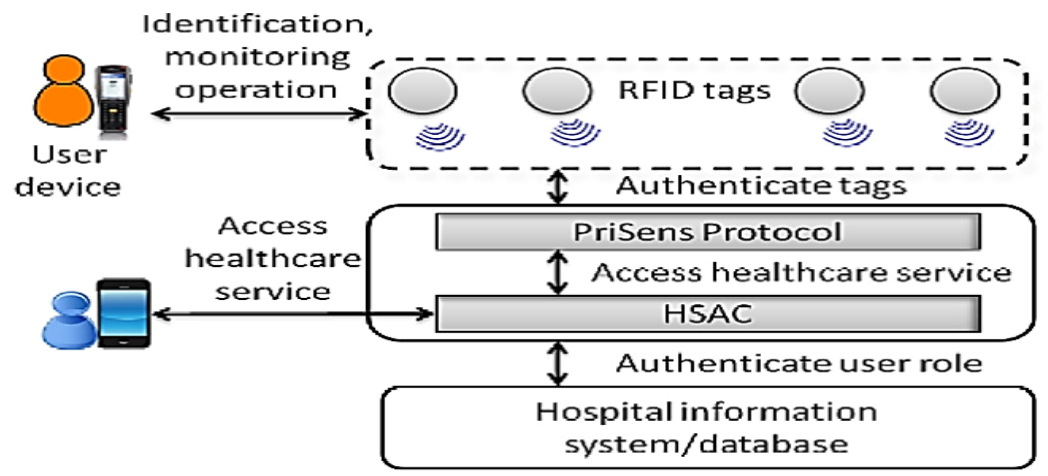

Fig 10: PriSens-HSAC framework architecture [의]

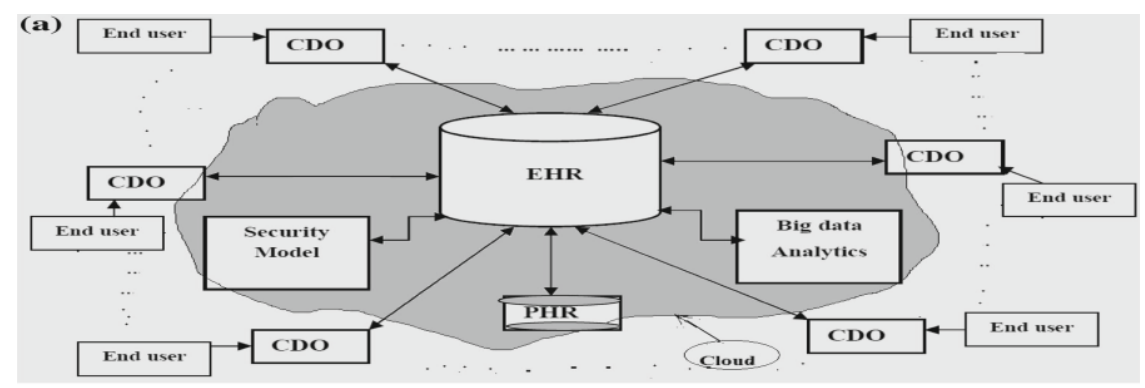

(b)

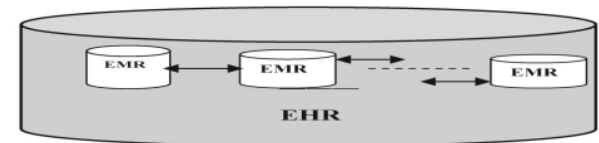

Fig 11: (a) Suggested framework for distributed health information system (b) A schematic of the EHR [무]

\section{Conceptual Framework for Big Data} Intelligent Health Care System

Pramanik et al. [41] have provided critical analysis of recent advances in health care systems (with emphasis on the application of smart technologies). They have proposed a conceptual framework for the big-data-driven smart healthcare system to provide more intelligent inclusive healthcare solutions. Some layers of this framework are (Fig 12):

\section{Data Source Layer:}

For managing structured, unstructured and semi- structured data sources.

\section{Data Analytics Layer:}

Performing computing, managing and visualizing big data.

\section{Smart Service Layer:}

Facilitating services such as: data monitoring, security and privacy agreements between consumer and service provider.

\section{Knowledge Discovery Layer:}


Add capabilities such as: anticipating needs for existence, planning and estimating, evaluating and modeling health care service mechanisms.

\section{Personalized big data framework for real- time health care preparation}

A big-data framework for real-time personalized health care customization based on field-based surveillance technology has been presented by Forkan et al. (Fig 13). This framework facilitates big data analysis within a cloud environment to detect patient-specific anomalies from large amounts of data. With the advent of the cloud, the big data generated is collected from heterogeneous fields and implements a two-step methodology [느].

The first step is to apply a Map-Reduce based Apriori algorithm to discover the relationship between field characteristics and threshold values of critical parameters of patient data.

Second, supervised learning algorithms, such as multilayer perceptron networks, decision trees, Bayesian networks, are implemented on the associative rules produced to implement contextaware decision making.

\section{A framework for evaluating health information technology}

Improving quality, efficiency and effectiveness of health services are the most important goals of health systems. If health organizations have the appropriate structure in place to regularly evaluate health information systems, they play an effective role in achieving these goals. The purpose of designing and implementing health information systems evaluation is to ensure the availability, use and applicability of data necessary to measure key health indicators.

Sadoughi et al. [43] described the process of evaluating health information systems, focusing on the WHO program. The stages of evaluating health information systems from the World Health Organization's perspective are as follows:

1) Determine the scope of authority

2) Collect and review existing relevant information

3) Finalize the evaluation services and topics

4) Determine system performance indicators
5) Designing and testing evaluation tools

6) Evaluation logistics

7) Performing evaluation

8) Analyze the results and prepare the evaluation report

9) Adjustment suggestions and preparation of the operation plan [푸 $]$

A conceptual framework for evaluating health information technology has been proposed by Eisenstein et al., Which can be used for hospitals, clinics, and health care systems [녀].

1) Domain: Specifies the difference between an information intervention and its achievement.

2) Mechanism: Specifies the specific components of the new information technology or health care system so that it can be the subject of an evaluation study.

3) Timing: Determines the occurrence before or after the implementation of health information technology.

The answers to these three questions determine a set of evaluation types, each comprising a set of evaluation questions, study designs, data collection requirements, and analysis methods.

\section{Framework for comprehensive health service evaluation and monitoring}

Reeve et al. [44] developed a framework for evaluating and monitoring a health care service, integrating hospital and community services. This framework comprises three main areas: structure, process, and outcome to determine health service performance (Fig 14).

\section{Development of frameworks for the semantic interaction of health information systems}

The major challenge for health information systems is the semantic interaction for establishing shared ehealth care. Lopez et al. [45] proposed a framework for the semantic interaction of health information systems. The proposed methodology is based on the Rational Unified Process (RUP) to integrate with other architectural approaches such as ServiceOriented Architecture (SOA), Model-Driven Architecture (MDA), ISO 10746, and HL7 Development Framework (HDF). The following Fig 15 shows the framework architecture. 


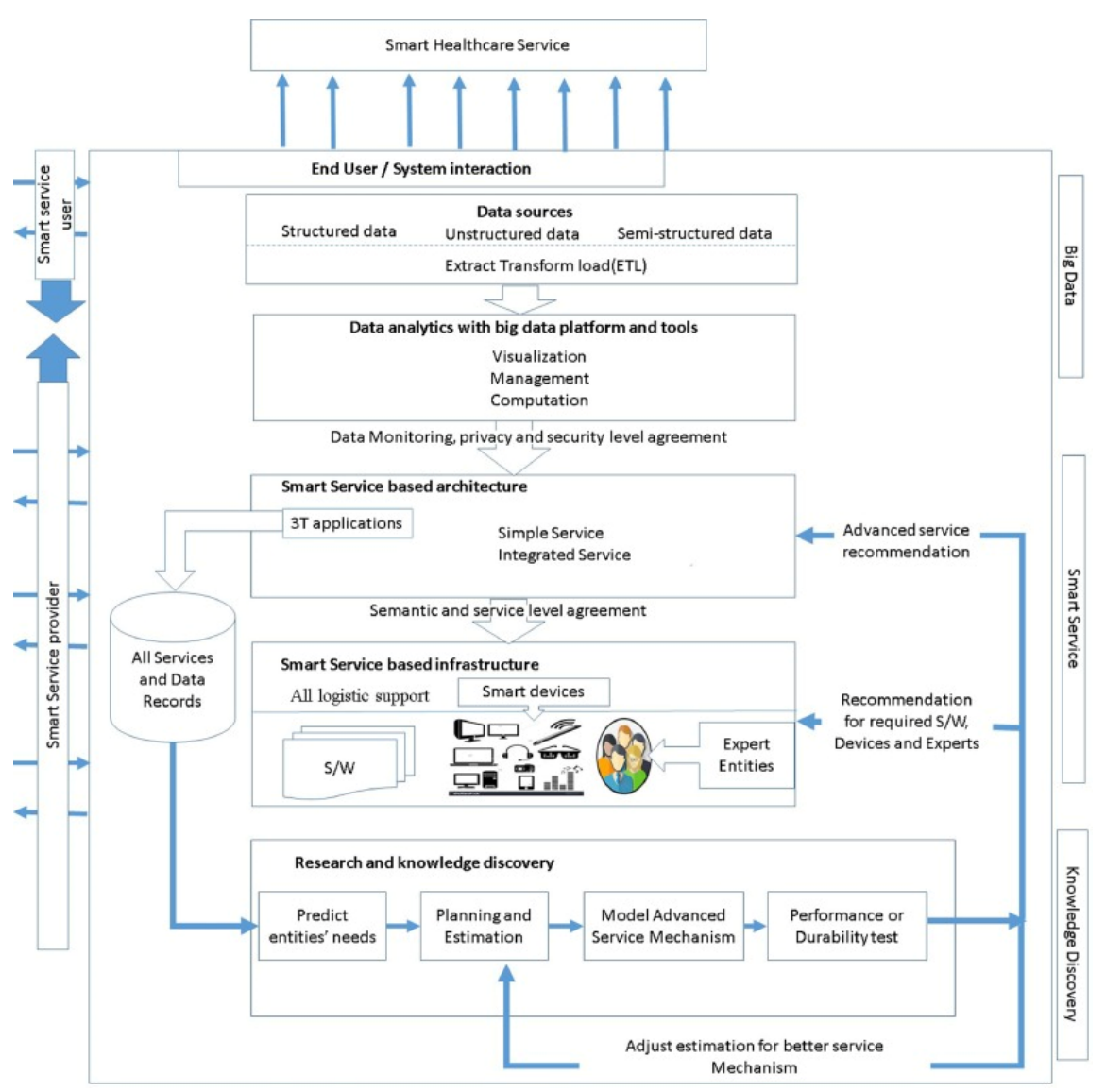

Fig 12: Applied conceptual framework for a health care system [41]

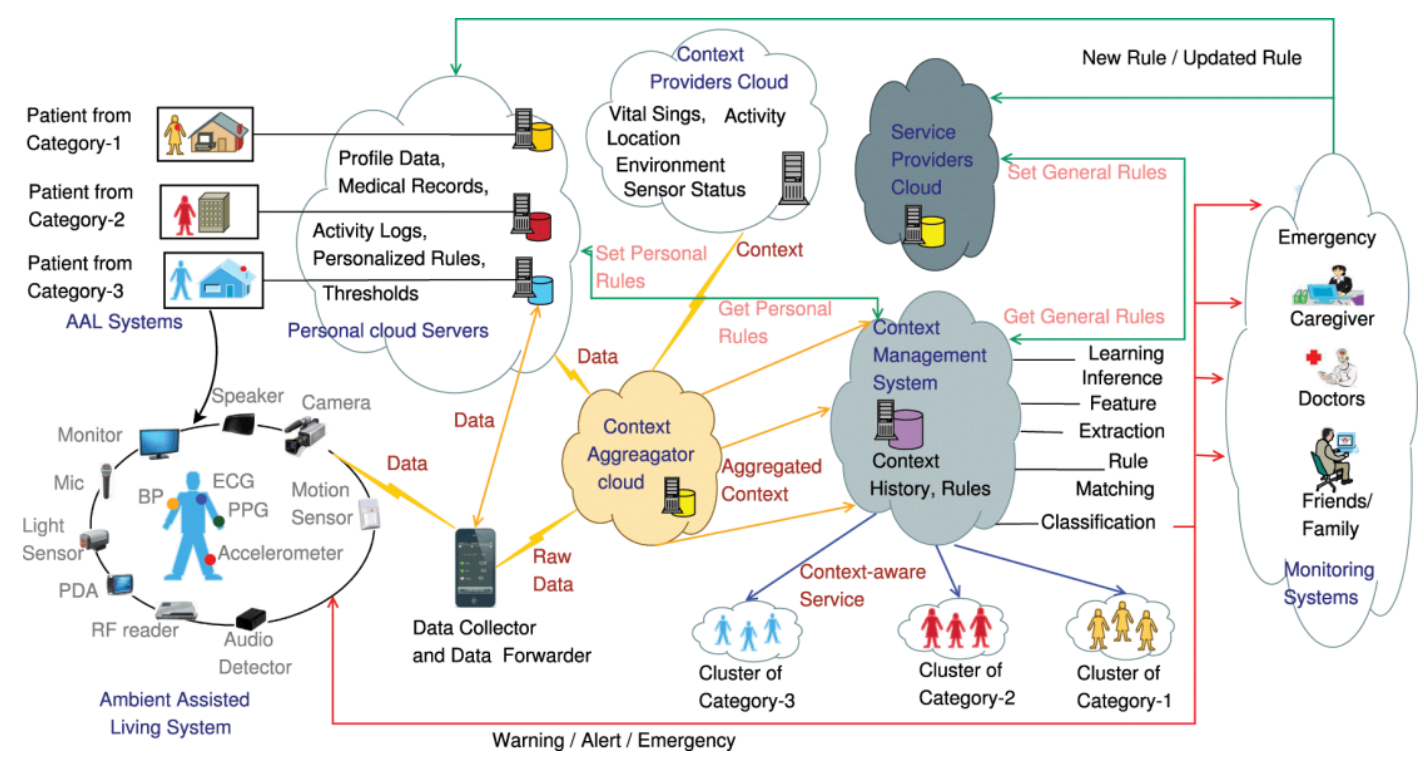

Fig 13: Full architecture of BDCaM model [르] 


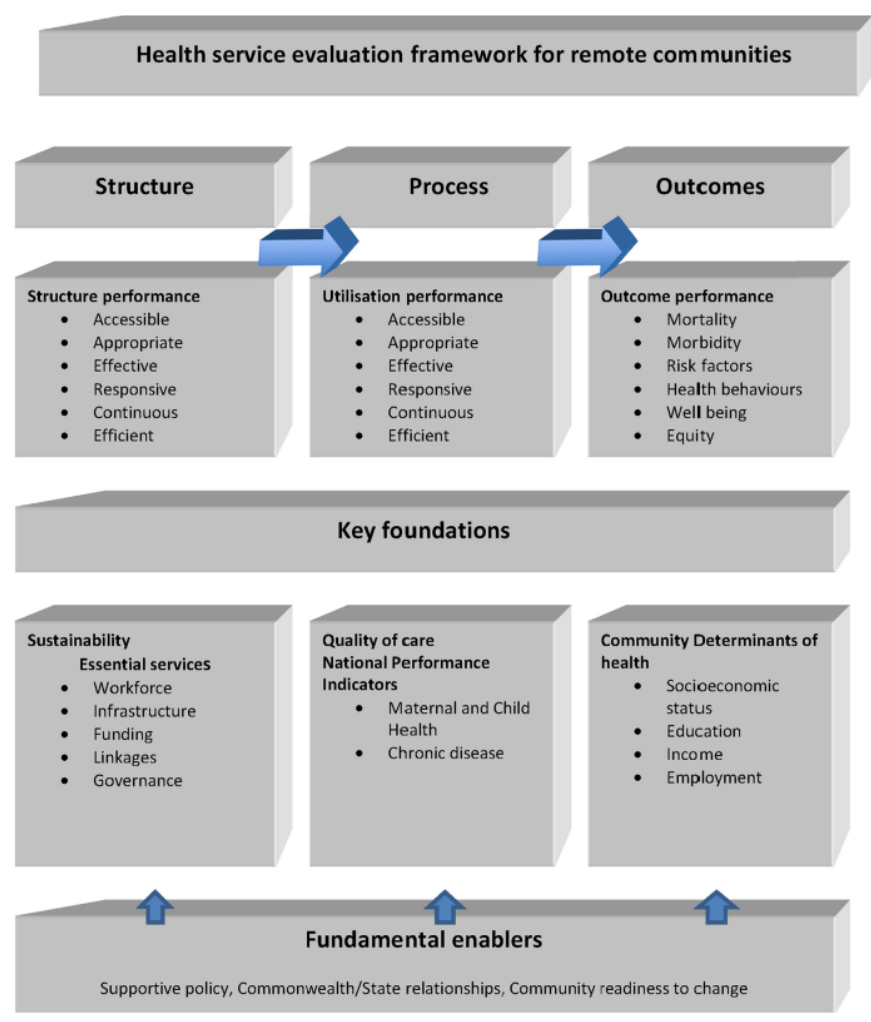

Fig 14: Health service evaluation framework [4]

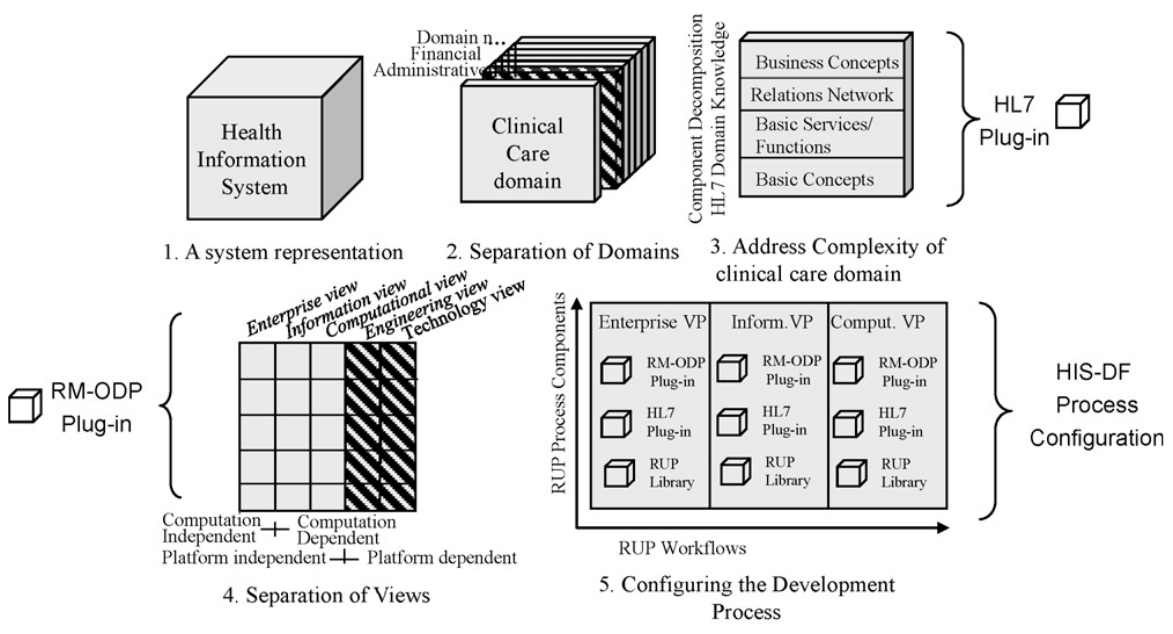

Fig 15: Framework for semantic interaction of health information systems [45]

\section{A framework for monitoring the health system}

In the global model of the health system, the role of governance and leadership is very important. Coordination between the various public and private actors in the utilization of health resources (labor, information, equipment and technology as well as the necessary funding) provides essential services to promote health and meet the needs of the community and fair participation in the provision of resources. Improving governance and leadership performance in the health system has an indispensable role in promoting health system functions such as fairness, efficiency and effectiveness and continuity in service delivery.

The model proposed by Mehrolhassani et al. [46] consists of 5 components:

Governance and Leadership:

A key component of the model, it regulates strategies and policies of other dimensions and areas of the health system.

\section{Provision of services:}

Includes different levels such as: primary health care, family physician, home care, health and 
hospital services, and rehabilitation

\section{Population Health:}

The situation is monitored for basic demographic indicators as well as mortality, disease burden and risk factors.

\section{Financial dimension:}

Examines the status of financing locations, resource accumulation, purchasing and resource specialization, payment system and strategic purchasing of services.

\section{Infrastructure:}

Examines the components of human resource management, knowledge and information, equipment and medicine as well as the legal nature of organizations.

Although these frameworks pursue specific health care goals, they are geared towards adopting standard architectural guidelines such as data collection, pre-processing, data analysis, interpretation and visualization. Due to the specific nature of the scope of the big data healthcare framework, professionals should make the utmost effort to select the tools used at different levels of the design and implementation of the framework $[\underline{47}, \underline{48}]$.

\section{DISCUSSION}

Various frameworks have been presented to date in the field of health care; for example, the framework for controlling and planning health care was proposed by Hall [무] , which the hospital managers used using the components of the framework, including resource management and principles Strategic counseling can be taken to improve care services. An applied conceptual framework of the health care system is also presented using big data analysis by Raghupathi and Raghupathi [을. This framework works for researchers who intend to use big data analyzes in the field of health as a road map. Rahman et al. [39] provided a RFID-based healthcare framework for addressing security issues. In this framework, two RFID tag-based authentication components and privacy have been considered as two important factors in maintaining security. Lopez et al. [45] introduced a framework for communicating between health information systems using the standards, protocols, and principles of interaction in these types of systems. Mehrolhassani et al. presented a framework for monitoring the health system, which can be used to evaluate a good health system, taking into account dimensions such as the financial dimension and delivery of services. Reeve et al. [44] developed a comprehensive evaluation and monitoring framework for a comprehensive health system that covers three areas of structure, process, and achievement. Chawla and Davis [31] suggested a personalized framework for the patient that the patient, using a mechanism based on the profiles of similar patients, predicted the risk for each patient. Considering the importance of focusing on the Big Data Area [49] and providing a lot of frameworks in this area, it is recommended that other important health care sectors provide frameworks in this regard, and it is also recommended in future studies of other widely used tools for software engineering in the field of health care Including modeling, simulations, etc., are being investigated, introduced and used [무] .

\section{CONCLUSION}

The framework is a valuable and widely used concept in the field of health care, which has been used in recent years. In this paper, the survey reviewed its implications in the field of health care. The framework in the field of health care has many advantages and especially for policy-makers to evaluate the system, measure and compare the function of health systems in different countries, for key decisions, the possibility of comparison within and between countries, identification Chatting and sharing of information. The results obtained on the basis of the framework can be used to manage health system investments, identify important fields of research in the field of health, as well as areas that are important and less worthwhile, and define new and useful research. The possibility of designing standard systems is provided.

\section{ACKNOWLEDGMENT}

The present study is the result of research project approved by the vice chancellery for research of Mashhad University of Medical Sciences (grant number 961731).

\section{AUTHOR'S CONTRIBUTION}

All the authors approved the final version of the manuscript.

\section{CONFLICTS OF INTEREST}

The authors declare no conflicts of interest regarding the publication of this study.

\section{FINANCIAL DISCLOSURE}

No financial interests related to the material of this manuscript have been declared.

\section{REFERENCES}

1. Framework [Internet]. 2019 [Cited: 15 May 2019]. Available 
https://www.dictionary.com/browse/framwork.

2. Introduction to Frameworks, Models \& Concepts [internet]. 2019 [cited: 5 Mar 2019]. Available from: http://www.odpedia.com/od/introduction-toframeworks-models-concepts/.

3. Stanojević V, Vlajić S, Milić M, Ognjanović M. Guidelines for framework development process. 7th Central and Eastern European Software Engineering Conference. IEEE; 2011.

4. Endter-Wada J, Blahna D, Krannich R, Brunson M. A framework for understanding social science contributions to ecosystem management. Ecological Applications. 1998; 8(3): 891-904.

5. Mandelblatt JS, Ramsey SD, Lieu TA, Phelps CE. Evaluating frameworks that provide value measures for health care interventions. Value Health. 2017; 20(2): 185-92. PMID: 28237193 DOI: 10.1016/j.jval.2016.11.013 [PubMed]

6. Olanrewaju RF, Islam T, Ali NA. An empirical study of the evolution of PHP MVC framework. Advanced Computer and Communication Engineering Technology. Springer; 2015.

7. Sinclair M. A guide to understanding theoretical and conceptual frameworks. Evidence-Based Midwifery. 2007; 5(2): 39-40.

8. Van Houdt S, Heyrman J, Vanhaecht K, Sermeus W, De Lepeleire J. An in-depth analysis of theoretical frameworks for the study of care coordination. Int J Integr Care. 2013; 13: 1-8. PMID: 23882171 [PubMed]

9. Petrini C. Theoretical models and operational frameworks in public health ethics. Int J Environ Res Public Health. 2010; 7(1): 189-202. PMID: 20195441 DOI: $10.3390 /$ ijerph7010189 [PubMed]

10. Ordooshahi M, Moghaddasian S, Lakdizaji S, Abdollahzadeh F, Mehdipour Zare N, Mohjal Aghdam $\mathrm{AR}$, et al. Management and research in nursing. Salemi Publication, Tehran; 2005.

11. Sicotte C, Champagne F, Contandriopoulos AP, Barnsley J, Béland F, Leggat S, et al. A conceptual framework for the analysis of health care organizations' performance. Health Serv Manage Res. 1998; 11(1): 24-41. PMID: 10178368 DOI: $10.1177 / 095148489801100106$ [PubMed]

12. Handler A, Issel M, Turnock B. A conceptual framework to measure performance of the public health system. Am J Public Health. 2001; 91(8): 1235-9. PMID: 11499110 [PubMed]

13. Murray CJ, Frenk J. A framework for assessing the performance of health systems. Bull World Health Organ. 2000; 78(6): 717-31. PMID: 10916909 [PubMed]

14. Huicho L, Dieleman M, Campbell J, Codjia L, Balabanova D, Dussault G, et al. Increasing access to health workers in underserved areas: A conceptual framework for measuring results. Bull World Health Organ. 2010; 88(5): 357-63. PMID: 20461135 DOI: 10.2471/BLT.09.070920 [PubMed]
15. Mosadeghrad AM. A conceptual framework for quality of care. Mater Sociomed. 2012; 24(4): 25161. $\quad$ PMID: 23922534 DOI: $10.5455 / \mathrm{msm} .2012 .24 .251-261$ [PubMed]

16. Khoja S, Durrani H, Scott RE, Sajwani A, Piryani U. Conceptual framework for development of comprehensive e-health evaluation tool. Telemed J E Health. 2013; 19(1): 48-53. PMID: 22957502 DOI: 10.1089/tmj.2012.0073 [PubMed]

17. Tyagi S, Agarwal A, Maheshwari P. A conceptual framework for IoT-based healthcare system using cloud computing. 6th International Conference of Cloud System and Big Data Engineering. IEEE; 2016.

18. Bourne PA. A theoretical framework of the good health status of Jamaicans: Using econometric analysis to model good health status over the life course. N Am J Med Sci. 2009; 1(2): 86-95. PMID: 22666677 [PubMed]

19. Fox A, Gardner G, Osborne S. A theoretical framework to support research of health service innovation. Aust Health Rev. 2015; 39(1): 70-5. PMID: 25513878 DOI: 10.1071/AH14031 [PubMed]

20. Arah OA, Klazinga NS, Delnoij DM, Asbroek AT, Custers T. Conceptual frameworks for health systems performance: A quest for effectiveness, quality, and improvement. Int J Qual Health Care. 2003; 15(5): 377-98. $\quad$ PMID: 14527982 DOI: 10.1093/intqhc/mzg049 [PubMed]

21. Affisco JF, Soliman KS. E-government: A strategic operations management framework for service delivery. Business Process Management Journal. 2006; 12(1): 13-21.

22. Araújo JR, José Luiz C, Maciel Filho R. Developing an operational framework for health policy analysis. Revista Brasileira de Saúde Materno Infantil. 2001; 1(3): 203-21.

23. Queensland clinical guidelines: Maternity and neonatal shared care [Internet]. 2016 [cited: 6 Mar 2019]. Available from: https://www.health.qld.gov.au/qcg/publications

24. Roe M, Malone S, Blake C, Collins K, Gissane C, Büttner F, et al. A six stage operational framework for individualising injury risk management in sport. Inj Epidemiol. 2017; 4(1): 26-32. PMID: 28929466 DOI: $10.1186 / \mathrm{s} 40621-017-0123-\mathrm{x}$ [PubMed]

25. Mei J, Liu H, Li X, Xie G, Yu Y. A decision fusion framework for treatment recommendation systems. Stud Health Technol Inform. 2015; 216: 300-4. PMID: 26262059 [PubMed]

26. Prasad S, Bruce LM, Ball JE. A multi-classifier and decision fusion framework for robust classification of mammographic masses. Conf Proc IEEE Eng Med Biol Soc. 2008; 2008: 3048-51. PMID: 19163349 DOI: 10.1109/IEMBS.2008.4649846 [PubMed]

27. Velikova M, Lucas PJ, Samulski M, Karssemeijer N. A probabilistic framework for image information fusion with an application to mammographic analysis. Medical Image Analysis. 2012; 16(4): 86575. 
28. Daunizeau J, Grova C, Marrelec G, Mattout J, Jbabdi S, Pélégrini-Issac $\mathrm{M}$, et al. Symmetrical event-related EEG/fMRI information fusion in a variational Bayesian framework. Neuroimage. 2007; 36(1): 6987. $\quad$ PMID: $17408972 \quad$ DOI: 10.1016/j.neuroimage.2007.01.044 [PubMed]

29. Raghupathi W, Raghupathi V. Big data analytics in healthcare: promise and potential. Health Inf Sci Syst. 2014; 2: 3-13. PMID: 25825667 doi: 10.1186/20472501-2-3 [PubMed]

30. Hall R. Handbook of healthcare system scheduling. Springer, US; 2011.

31. Chawla, NV, Davis DA. Bringing big data to personalized healthcare: A patient-centered framework. J Gen Intern Med. 2013; 28 (Suppl 3): S660-5. PMID: 23797912 DOI: 10.1007/s11606-0132455-8 [ubMed]

32. Kim T, Park K, Yi S, Kim H. A big data framework for u-healthcare systems utilizing vital signs. International Symposium on Computer, Consumer and Control. IEEE; 2014.

33. Fang R, Pouyanfar S, Yang, Chen SC, Iyengar SS. Computational health informatics in the big data age: A survey. ACM Computing Surveys. 2016; 49(1): 12.

34. Youssef AE. A framework for secure healthcare systems based on big data analytics in mobile cloud computing environments. Int J Ambient Syst Appl. 2014; 2(2): 1-11.

35. del Carmen Legaz-García M, Martínez-Costa M, Menárguez-Tortosaa $\mathrm{M}$, Fernández-Breisa JT. A semantic web based framework for the interoperability and exploitation of clinical models and EHR data. Knowledge-Based Systems. 2016; 105: 175-89.

36. Sakr S, Elgammal A. Towards a comprehensive data analytics framework for smart healthcare services. Big Data Research. 2016; 4: 44-58.

37. Mahmud S, Iqbal R, Doctor F. Cloud enabled data analytics and visualization framework for healthshocks prediction. Future Generation Computer Systems. 2016; 65: 169-81.

38. Jokonya 0 . Towards a big data framework for the prevention and control of HIV/AIDS, TB and Silicosis in the mining industry. Procedia Technology. 2014; 16: $1533-41$.

39. Rahman F, Bhuiyan MZA, Ahamed SI. A privacy preserving framework for RFID based healthcare systems. Future Generation Computer Systems. 2017; 72: 339-52.

40. Sarkar BK. Big data for secure healthcare system: A conceptual design. Complex \& Intelligent Systems. 2017; 3(2): 133-51.

41. Pramanik MI, Lau RYK, Demirkan H, Kalam Azad MA. Smart health: Big data enabled health paradigm within smart cities. Expert Systems with Applications. 2017; 87: 370-83.

42. Forkan ARM, Khalil I, Ibaida A, Tari Z. BDCaM: Big data for context-aware monitoring- A personalized knowledge discovery framework for assisted healthcare. IEEE Transactions on Cloud Computing. 2017; 5(4): 628-41.

43. Sadoughi F, Aminpour F. How to evaluate health information systems: Evaluation stages. Iranian Journal of Medical Education. 2011; 10(5): 950-63.

44. Eisenstein EL, Juzwishin D, Kushniruk AW, Nahm M. Defining a framework for health information technology evaluation. Stud Health Technol Inform. 2011; 164: 94-9. PMID: 21335694 [PubMed]

45. Lopez DM, Blobel BG. A development framework for semantically interoperable health information systems. Int J Med Inform. 2009; 78(2): 83-103. PMID: $18621574 \quad$ DOI: 10.1016/j.ijmedinf.2008.05.009 [PubMed]

46. Mehrolhassani M, Haghdoost AA, Dehnavieh $\mathrm{R}$, Abolhallaje M, Emami M. The proposed framework for monitoring health system. Iranian Journal of Epidemiology. 2017; 12: 92-6.

47. Chen CLP, Zhang CY. Data-intensive applications, challenges, techniques and technologies: A survey on big data. Information Sciences. 2014; 275: 314-47.

48. Sukumar SR, Natarajan R, Ferrell RK. Quality of big data in health care. Int J Health Care Qual Assur. 2015; 28(6): 621-34. PMID: 26156435 DOI: 10.1108/IJHCQA-07-2014-0080 [ubMed]

49. Nazari E, Shahriari MH, Tabesh H. Big data analysis in healthcare: Apache Hadoop, Apache spark and Apache Flink. Front Health Inform. 2019; 8(1): e14.

50. National Academy of Engineering, Institute of Medicine Committee on Engineering, the Health Care System. The national academies collection: Reports funded by national institutes of health. In: Reid PP, Compton WD, Grossman JH, Fanjiang G (ed). Building a better delivery system: A new engineering/health care partnership. Washington DC: National Academies Press (US); 2005. 